\title{
Action Transformations in the ACTRESS Compiler Generator
}

\author{
Hermano Moura ${ }^{\star}$, David A. Watt ${ }^{\star}$
}

\begin{abstract}
Given the action-semantic description of a source language, ACTRESS generates a compiler. The generated compiler translates its source program first to an action, and then to object code. Transformations of the intermediate action greatly improve the efficiency of the object code. This paper studies these transformations.
\end{abstract}

\section{Introduction}

ACTRESS [2] is an action-semantics directed compiler generator. That is to say, it accepts a formal description of the syntax and action semantics of a particular programming language, the source language, and from this it automatically generates a compiler that translates the source language to $\mathrm{C}$ object code. The generated compiler translates each source program to an action, which we call the program action, and thereafter translates the program action to object code.

We have used ACTRESS to generate compilers for a small functional language, Mini-ML, and a small imperative language, Mini- $\triangle$. The preliminary version of ACTREss[2] generated compilers whose object code was rather inefficient. However, significant improvements have since been achieved.

Inspection of typical program actions reveals many opportunities for simplification. For example, actions obey simple algebraic laws [7], which can be exploited to simplify them. However, more sophisticated transformations are needed to achieve major simplifications. Our recent work has been directed to discovering, formalising, and implementing such transformations. Our ultimate aim is to make ACTRESS generate compilers whose object code is only about 2-4 times slower than that of hand-crafted compilers.

This paper describes our recent work. The rest of the paper is structured as follows. Section 2 is a very brief summary of the ACTRESS subset of action notation. Section 3 is a brief description of ACTREss itself. Section 4 motivates the need for action transformations in generated compilers, focusing on algebraic simplification, transient elimination, binding elimination, and storage allocation. Section 5 shows how we formalise these transformations. Section 6 outlines how we implement them, and includes a worked example of action transformations in the generated MiNI- $\triangle$ compiler. Section 7 concludes. Appendix A outlines an

\footnotetext{
* Caixa Econômica Federal, Brazil. E-mail: hermano@di.ufpe.br. Work supported by CNPq, Brazil.

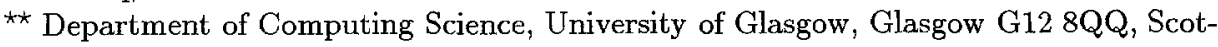
land. E-mail; dawedcs.glasgow.ac.uk.
} 
action-semantic description of MiNI- $\triangle$, which we use as a running example in this paper.

A fuller version of this paper is available as [10].

\section{Action Notation}

Action semantics was developed by Mosses and Watt $[7,8,16]$. Action semantics has very good pragmatic qualities: language descriptions are unusually easy to read, to write, and to modify.

Action notation provides a number of action primitives, action combinators, and yielders. An action primitive represents a single computational step, such as giving a datum (transient), binding an identifier to a datum, or storing a datum in a cell. An action combinator combines sub-actions into a composite action, and governs the control flow and the data flow (transients, bindings, and storage) between these sub-actions. There are action combinators that correspond to sequential composition, functional composition, choice, iteration, and so on. Finally, some primitive actions include yielders, which are used to access data flowing into the action. Some of the more common action primitives, action combinators, and yielders are summarised in Table 1.

An action-semantic description of a programming language $\mathcal{L}$ specifies a mapping from the phrases of $\mathcal{L}$ to action notation. An action-semantic description is structured like a denotational description, with semantic functions and semantic equations, but the denotations of phrases are expressed in action notation. Extracts from an action-semantic description of MinI- $\Delta$ may be found in Appendix A.

\section{The ACTREss Compiler Generator}

ACTRESS is a compiler generation system developed at the University of Glasgow by Brown, Moura, and Watt [2]. It provides a collection of modules operating on actions (represented internally as trees). These modules include the following.

Check $k_{\mathcal{A}}$ is the action notation sort checker. ${ }^{3}$ This infers the sort of a given action and each of its sub-actions. It annotates each action with its sort.

Encode $_{\mathcal{A}}$ is the action notation code generator. This translates the (annotated) action to $\mathrm{C}$ object code.

Other modules are generated by ACTREss from the formal description of a particular source language $\mathcal{L}$.

Parse $_{\mathcal{L}}$ is a parser for $\mathcal{L}$. This parser is generated from a syntactic description of $\mathcal{L}$, syntax $\mathcal{L}$, using the standard parser generator, mlyacc:

$$
\operatorname{parse}_{\mathcal{L}}=\operatorname{mlyacc}\left(\operatorname{syntax}_{\mathcal{L}}\right)
$$

3 A sort in action semantics is roughly equivalent to a type. The sort of an action includes the sorts of all transients and bindings passed into and out of that action. 


\begin{tabular}{|c|c|}
\hline Primitive action & Meaning \\
\hline complete & Completes immediately (i.e., does nothing). \\
\hline fail & Fails immediately. \\
\hline give $Y$ & Gives the datum yielded by $Y$, labelled 0 . \\
\hline give $Y$ label $\# n$ & Gives the datum yielded by $Y$, labelled $n$. \\
\hline bind $k$ to $Y$ & $\begin{array}{l}\text { Produces a single binding, of identifier } k \text { to the datum yielded by } \\
Y \text {. }\end{array}$ \\
\hline $\begin{array}{l}\text { recursively } \\
\text { bind } k \text { to } Y\end{array}$ & As 'bind', but allows the binding of $k$ to be used in evaluating $Y$. \\
\hline store $Y_{1}$ in $Y_{2}$ & Stores the datum yielded by $Y_{1}$ in the cell yielded by $Y_{2}$. \\
\hline allocate a $S$ & Finds an unreserved cell of sort $S$, reserves it, and gives it. \\
\hline enact $Y$ & Performs the action incorporated by the abstraction yielded by $Y$. \\
\hline Composite action & Meaning \\
\hline$A_{1}$ or $A_{2}$ & $\begin{array}{l}\text { Performs either } A_{1} \text { or } A_{2} \text {. If the chosen sub-action fails, the other } \\
\text { sub-action is chosen. }\end{array}$ \\
\hline$A_{1}$ else $A_{2}$ & $\begin{array}{l}\text { Tests the given truth value, and then performs } A_{1} \text { if it is true or } \\
A_{2} \text { if it is false. }\end{array}$ \\
\hline unfolding $A$ & $\begin{array}{l}\text { Performs } A \text {. Dummy action 'unfold', whenever encountered inside } \\
A, \text { is replaced by } A \text {. Used for iteration. }\end{array}$ \\
\hline$A_{1}$ and $A_{2}$ & $\begin{array}{l}\text { Performs both } A_{1} \text { and } A_{2} \text { collaterally. Any transients given by } A_{1} \\
\text { and } A_{2} \text { are merged. Any bindings produced by } A_{1} \text { and } A_{2} \text { are } \\
\text { merged. }\end{array}$ \\
\hline$A_{1}$ and then $A_{2}$ & $\begin{array}{l}\text { Performs } A_{1} \text { and } A_{2} \text { sequentially. Otherwise behaves like ' } A_{1} \text { and } \\
A_{2} \text { '. }\end{array}$ \\
\hline$A_{1}$ then $A_{2}$ & $\begin{array}{l}\text { Performs } A_{1} \text { and } A_{2} \text { sequentially. Transients given by } A_{1} \text { are given } \\
\text { to } A_{2} \text {. }\end{array}$ \\
\hline$A_{1}$ hence $A_{2}$ & $\begin{array}{l}\text { Performs } A_{1} \text { and } A_{2} \text { sequentially. Bindings produced by } A_{1} \\
\text { propagate to } A_{2} \text {. }\end{array}$ \\
\hline$A_{1}$ before $A_{2}$ & $\begin{array}{l}\text { Performs } A_{1} \text { and } A_{2} \text { sequentially. Bindings produced by } A_{1} \text { and } \\
A_{2} \text { are accumulated. }\end{array}$ \\
\hline furthermore $A$ & Performs $A$. Bindings produced by $A$ override received bindings. \\
\hline Yielder & Meaning \\
\hline the $S$ & The given transient datum labeled 0 . It must be of sort $S$. \\
\hline the $S \# n$ & The given transient datum labeled $n$. It must be of sort $S$. \\
\hline the $S$ bound to $k$ & The datum currently bound to identifier $k$. It must be of sort $S$. \\
\hline the $S$ stored in $Y$ & $\begin{array}{l}\text { The datum currently contained in the cell yielded by } Y \text {. It must } \\
\text { be of sort } S \text {. }\end{array}$ \\
\hline abstraction $A$ & The abstraction that incorporates action $A$. \\
\hline closure $Y$ & $\begin{array}{l}\text { The abstraction yielded by } Y \text {, with the current bindings supplied } \\
\text { to the incorporated action. }\end{array}$ \\
\hline$Y_{1}$ with $Y_{2}$ & $\begin{array}{l}\text { The abstraction yielded by } Y_{1} \text {, with the transient datum yielded } \\
\text { by } Y_{2} \text { supplied to the incorporated action. }\end{array}$ \\
\hline
\end{tabular}

Table 1. Some primitive actions, composite actions, and yielders. 
Actioneer $_{\mathcal{L}}$ is a module that translates a parsed $\mathcal{L}$ program to the corresponding program action. This module is generated from a semantic description of $\mathcal{L}$, semantics $\mathcal{L}_{\mathcal{L}}$, using the actioneer generator, actgen:

$$
\text { actioneer }_{\mathfrak{L}}=\operatorname{actgen}\left(\text { semantics }_{\mathcal{L}}\right)
$$

The actioneer generator treats semantics $\mathcal{L}_{\mathcal{L}}$ as a syntax-directed translation from $\mathcal{L}$ to action notation.

Composition of the generated parser and actioneer for $\mathcal{L}$ with the action notation sort checker and code generator yields a compiler for language $\mathcal{L}$ :

$$
\text { compile }_{\mathcal{L}}=\text { encode }_{\mathcal{A}} \circ \text { check }_{\mathcal{A}} \circ \text { actioneer }_{\mathcal{L}} \circ \text { parse }_{\mathcal{L}}
$$

We have recently added a new module to ACTRESS. Transform $m_{\mathcal{A}}$ is the action transformer, which attempts to simplify a given (annotated) action. This module may be used to construct a compiler that generates more efficient object code:

$$
\text { compile }_{\mathcal{L}}^{\prime}=\text { encode }_{\mathcal{A}} \circ \text { transform }_{\mathcal{A}} \circ \text { check }_{\mathcal{A}} \circ \text { actioneer }_{\mathcal{L}} \circ \text { parse }_{\mathcal{L}}
$$

\section{Action Transformations}

When a source program is translated to a program action, the latter is generated by piecing together fragments of action notation from different semantic equations in the action-semantic description. Examination of the program action tends to reveal many opportunities for simplification. In this section we introduce some of the more common possibilities.

\section{Algebraic Simplification}

Actions enjoy a variety of nice algebraic properties [7]. For example: 'and then' is associative, and has 'complete' as a unit; 'or' is associative, commutative, and idempotent, and has 'fail' as a unit.

These algebraic laws are used by the sort checker and transformer to simplify progam actions. Consider the following MiNI- $\triangle$ command:

let $\operatorname{var} n:$ int in $\ldots n$

The command is mapped to the following action:

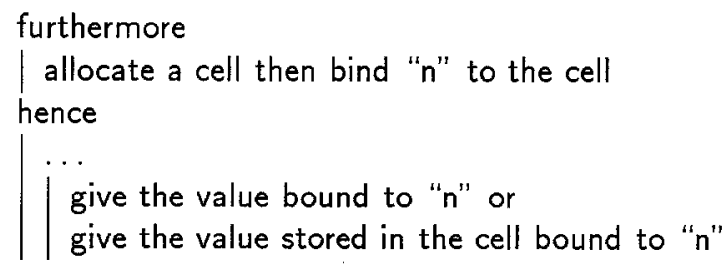

The sub-action on the last two lines is 'evaluate 【" $n$ "】' - see semantic equation A.2(6). In the context of the above action, the sort checker infers that the subaction receives a binding of identifier " $n$ " to a cell. It follows that "give the value bound to " $n$ "' must fail (since a cell is not a value) and can be replaced by 'fail': 
give the value stored in the cell bound to " $\mathrm{n}$ "

Now we can apply algebraic simplification, 'fail' being a unit of 'or':

$\mid \begin{aligned} & \text { give the value stored in the cell bound to " } n \text { " } \\ & \text { give }\end{aligned}$

In fact, the sub-actions 'give the value bound to $I$ ' and 'give the value stored in the cell bound to $I^{\prime}$ in equation A.2(6) are mutually exclusive, since the sorts value and cell are mutually disjoint. At least one of the sub-actions must fail. This point is important because it allows ACTRESS to infer automatically that this 'or' corresponds to a static (compile-time) choice.

In addition to any opportunities for algebraic simplification in the original program action, such as the above, we shall see that other action transformations tend to expose further opportunities for algebraic simplification.

\section{Transient Elimination}

Program actions often contain 'give' actions that can easily be eliminated. For example, the MINI- $\Delta$ constant declaration "const $n \sim 10$ " is mapped by semantic equations A.2(5, 8$)$ to the following action:

give 10 then bind " $n$ " to the value

It should be clear that we can simplify the action to:

bind " $n$ " to 10

This is an example of transient elimination.

\section{Binding Elimination}

A typical program action contains many 'bind' actions, arising from the translation of declarations, and other actions that use these bindings. Immediate translation of the program action generates object code that explicitly manipulates sets of bindings - a large overhead. Fortunately, we can avoid this overhead in most cases, using an action transformation called binding elimination.

Consider the following action:

bind " $x$ " to 7 hence ... the value bound to " $x$ "

The sub-action 'bind " $x$ " to 7 ' produces a binding of the identifier " $x$ " to the datum 7. The scope of this binding is the sub-action following 'hence'. Within this sub-action, the yielder 'the value bound to " $x$ "' is an applied occurrence of the identifier " $x$ ". Clearly, this yielder will yield 7 whenever it is evaluated, so we can simply replace it by ' 7 '. Now the 'bind' action becomes redundant, and we can replace it by 'complete': 
complete hence ... 7

This action can now be algebraically simplified.

Binding elimination can also be applied in more complicated situations. Consider the following action:

bind " $y$ " to the value hence

... the value bound to " $y$ "

which might be the body of an abstraction (which is given an argument value). The sub-action 'bind " $y$ " to the value' produces a binding of the identifier " $y$ " to a (statically) unknown datum - all we know about this datum is that it must be of sort value. Thus we cannot simply replace applied occurrences of " $y$ " by the datum to which " $y$ " is bound. What we can do is to store the unknown datum in a known cell, say cell $(1,4)^{4}$, and replace each applied occurrence of " $y$ " by a fetch from that cell:

store the value in cell $(1,4)$ hence

$\ldots$ the value stored in cell $(1,4)$

We define an action to be statically scoped if, for every applied occurrence of an identifier $k$ in the action, we can uniquely identify the 'bind' action that produced the current binding of $k$.

We define a programming language to be statically scoped if every program in the language is mapped to an action that is statically scoped. Otherwise the programming language is dynamically scoped.

Action semantics is quite capable of specifying the semantics of both statically and dynamically scoped languages. In general, binding elimination will be fully effective in program actions generated from statically scoped languages, but only partly effective in those generated from dynamically scoped languages.

Moura's thesis [9] proposes the following sufficient condition for static-scopedness. A language $\mathcal{L}$ is statically scoped if in an action-semantic description of $\mathcal{L}:$

- 'abstraction $A$ ' always occurs in the context 'closure (abstraction $A$ )';

- in any occurrence of 'abstraction $A$ ', 'unfolding $A$ ', or ' $A_{1}$ or $A_{2}$ ', the subactions $A, A_{1}$, and $A_{2}$ produce no bindings.

This condition is of theoretical interest because it offers, for the first time as far as we know, a definition of static-scopedness related to the programming language's formal description. It is also of practical interest because it will be used in a future version of ACTRESS. If ACTRESS finds the source language to be statically scoped, it will build that useful knowledge into the generated compiler and its run-time system. (Even if it finds the source language to be dynamically scoped, ACTRESS will still generate a compiler, but the overhead of manipulating sets of bindings at run-time will, inevitably, still be there.)

4 This notation will be explained later. 


\section{Static Allocation}

Another important transformation is concerned with storage allocation. The action 'allocate a cell' finds and reserves an unused cell; in other words, it is dynamic allocation. This is in fact the only form of storage allocation provided by standard action notation. ${ }^{5}$

As is well known, however, storage space for global and local variables can be more efficiently allocated in frames (or activation records). The basis of such static allocation ${ }^{6}$ is as follows. All the variables local to a particular procedure $P$ are allocated together in a frame, which is essentially a sequence of contiguous cells, and the position $q$ of each variable within the frame is statically known. The static nesting level $l$ of $P$ is also known. (For the main program, $l=0$.) At run-time, the $l$ th display register points to the frame within which these local variables have been allocated, whenever $P$ is active and these variables are accessible. Thus the address of each local variable is completely determined by the pair $(l, q)$, which is statically known.

In the context of action notation, for 'procedure' read 'abstraction', and for 'main program' read 'program action'. For the purposes of the transformation about to be described, we extend action notation with a non-standard yielder, cell $(l, q)$. When evaluated at run-time, this will yield the $q$ th cell in the frame currently at display level $l$.

The Mini- $\triangle$ command "let var $v$ : bool in $v:=$ true" is mapped to the following action:

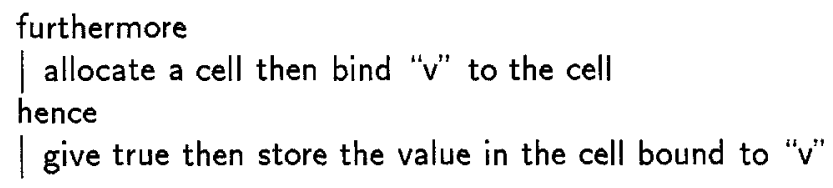

Suppose, for the moment, that this action is part of the program action, i.e., it is at level 0 . Instead of dynamically allocating a cell, we can statically allocate a known cell, say cell $(0,5)$, and transform the action to:

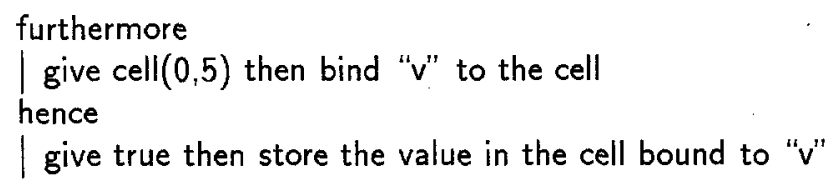

Now we can apply transient and binding elimination, taking advantage of the fact that " $v$ " is bound to a known cell:

furthermore complete

hence store true in cell $(0,5)$

\footnotetext{
${ }^{5}$ Action notation could be enhanced with explicit static allocation, but that would make the notation more complicated, harder to formalise, and more implementationoriented.

${ }^{6}$ Some authors use static allocation more restrictively, to mean the allocation of fixed addresses to global variables. In this paper, however, we shall use the same term to include allocation of frame-based addresses to local variables.
} 
If the same action occurs as the body of an abstraction, i.e., at a level greater than 0 , similar transformations are still possible.

Not all 'allocate' actions can be transformed in this way. Some source language constructs demand dynamic allocation, an example being the new allocator in PASCAL. So we must be able to distinguish between static and dynamic allocations.

Consider an 'allocate' action contained in action $A$, where $A$ is either the program action or the body of an abstraction. Let $l$ be the static nesting level of $A$. Then:

- If this 'allocate' action occurs in a static context within $A$, replace the 'allocate' action by 'give cell $(l, q)$ ', choosing $q$ such that cell $(l, q)$ is not used for any other purpose in $A$.

In action notation it is rather easy to distinguish between static and dynamic contexts within $A$. Basically, sub-actions of the combinators 'or', 'else', and 'unfolding' are in dynamic contexts; all other sub-actions are in static contexts. This is, admittedly, a fairly crude test, although always erring on the side of safety.

\section{Formalisation}

Having motivated the principal action transformations in Section 4, our next step is to formalise them. We shall do this by means of inference rules. To formalise an action transformation we use the judgment:

$$
\mathcal{K}, \mathcal{T}, \mathcal{B}, \mathcal{S} \vdash A \sim A^{\prime}, \mathcal{T}^{\prime}, \mathcal{B}^{\prime}, \mathcal{S}^{\prime}
$$

where:

- $A$ is the original action, and $A^{\prime}$ is the transformed action.

- $\mathcal{K}$ is sort information, from which the sort of $A$ can be determined.

- $\mathcal{T}$ and $\mathcal{T}^{\prime}$ are transient substitutions applicable to the transients flowing into and out of $A$, respectively. The transient substitution:

$$
\mathcal{T}=\left[d / n, d^{\prime} / n^{\prime}, \ldots\right]
$$

indicates that 'the $S \# n$ ' is to be replaced by the known datum $d$, 'the $S$ $\# n^{\prime \prime}$ is to be replaced by the known datum $d^{\prime}$, and so on.

$-\mathcal{B}$ and $\mathcal{B}^{\prime}$ are binding substitutions applicable to the bindings flowing into and out of $A$, respectively. The binding substitution:

$$
\mathcal{B}=\left[d / k, \text { the }- \text { stored in } c^{\prime} / k^{\prime}, \ldots\right]
$$

indicates that a scoped applied occurrence of 'the $S$ bound to $k$ ' is to be replaced by the known datum $d$, a scoped applied occurrence of 'the $S$ bound to $k^{\prime}$ ' is to be replaced by 'the $S$ stored in $c^{\prime}$ ', and so on. 
$\mathcal{S}$ and $\mathcal{S}^{\prime}$ are the storage allocation contexts before and after transforming $A$, respectively. The storage allocation context:

$$
\mathcal{S}=(l, q, s d)
$$

indicates that action $A$ is at scope level $l$, that $q$ cells have been allocated statically in the current scope, and that $s d$ indicates whether $A$ is in a it static or it dynamic context.

Similarly, to formalise a yielder transformation, we use the judgment:

$$
\mathcal{K}, \mathcal{T}, \mathcal{B}, \mathcal{S} \vdash Y \sim Y^{\prime}
$$

\section{Binding Elimination}

Binding elimination is formalised by the following inference rules:

$$
\frac{\mathcal{K} \vdash \text { bind } k \text { to } Y:(\tau, \beta) \hookrightarrow(\{\},\{k: d\})}{\mathcal{K}, \mathcal{T}, \mathcal{B}, \mathcal{S} \vdash \text { bind } k \text { to } Y \sim \text { complete, }[],[d / k], \mathcal{S}}
$$

$$
\begin{aligned}
& \mathcal{K} \vdash \text { bind } k \text { to } Y:(\tau, \beta) \hookrightarrow(\{\},\{k: S\}) \quad \mathcal{K}, \mathcal{T}, \mathcal{B}, \mathcal{S} \vdash Y \leadsto Y^{\prime} \\
& \hline \mathcal{K}, \mathcal{T}, \mathcal{B},(l, q, \text { stat }) \vdash \text { bind } k \text { to } Y \leadsto \\
& \text { store } Y^{\prime} \text { in cell }(l, q),[],[\text { the } \text { - stored in cell }(l, q) / k],(l, q+1, \text { stat })
\end{aligned}
$$

$$
\overline{\mathcal{K}, \mathcal{T},[d / k, \ldots], \mathcal{S} \vdash \text { the } S \text { bound to } k \leadsto \text { the } S \text { yielded by } d}
$$

$$
\begin{aligned}
& \overline{\mathcal{K}, \mathcal{T} \text {, [the - stored in } c / k, \ldots], \mathcal{S} \vdash \text { the } S \text { bound to } k \leadsto \text { the } S \text { stored in } c} \\
& \frac{\mathcal{K}, \mathcal{T}, \mathcal{B}, \mathcal{S} \vdash A_{1} \leadsto A_{1}^{\prime}, \mathcal{T}_{1}^{\prime}, \mathcal{B}_{1}^{\prime}, \mathcal{S}_{1}^{\prime} \quad \mathcal{K}, \mathcal{T}, \mathcal{B}_{1}^{\prime}, \mathcal{S}_{1}^{\prime} \vdash A_{2} \leadsto A_{2}^{\prime}, \mathcal{T}_{2}^{\prime}, \mathcal{B}_{2}^{\prime}, \mathcal{S}_{2}^{\prime}}{\mathcal{K}, \mathcal{T}, \mathcal{B}, \mathcal{S} \vdash A_{1} \text { hence } A_{2} \leadsto A_{1}^{\prime} \text { hence } A_{2}^{\prime}, \mathcal{T}_{1}^{\prime} \oplus \mathcal{T}_{2}^{\prime}, \mathcal{B}_{2}^{\prime}, \mathcal{S}_{2}^{\prime}}
\end{aligned}
$$

Rule (10) applies when the action 'bind $k$ to $Y$ ' binds identifier $k$ to a known datum $d .^{7}$ The 'bind' action is replaced by 'complete', and the output binding substitution contains the information that scoped applied occurrences of 'the $S$ bound to $k^{\prime}$ are to be replaced by $d$. Rule (12) formalises the latter transformation.

Rule (11) applies when the action 'bind $k$ to $Y$ ' binds identifier $k$ to an unknown datum. In (11), The 'bind' action is replaced by 'store $Y$ ' in cell $(l, q)$ ', and the output binding substitution contains the information that scoped applied occurrences of 'the $S$ bound to $k$ ' are to be replaced by 'the $S$ stored in cell $(l, q)$ '. Rule (13) formalises the latter transformation. Rule (11) takes $(l, q)$ from the storage allocation context, in the same way as (15) discussed below.

Rule (14) links together rules $(10-11)$ and $(12-13)$. In the action ' $A_{1}$ hence $A_{2}$ ', the bindings produced by $A_{1}$ are propagated into $A_{2}$. Therefore, $A_{1}$ 's output binding substitution $\mathcal{B}_{1}^{\prime}$ becomes $A_{2}$ 's input binding substitution.

\footnotetext{
${ }^{7}$ This case and that of rule (11) are distinguished by whether the sort of the 'bind' action associates $k$ to an individual $d$ or to a proper sort $S$.
} 


\section{Static Allocation}

Static allocation is formalised by the following inference rule:

$$
\overline{\mathcal{K}, \mathcal{T}, \mathcal{B},(l, q, \text { stat }) \vdash \text { allocate a cell } \sim \text { give cell }(l, q),[],[],(l, q+1, \text { stat })}
$$

Rule (15) applies when the action 'allocate a cell' occurs in a static context, where it is safe to allocate a known address $(l, q)$. Here $(l, q)$ is taken from the input storage allocation context. In the output storage allocation context the cell count is incremented to $q+1$.

When we transform the program action, we take its input storage allocation context to be $(0,0$, stat $)$. When we transform the body $A$ of an abstraction 'abstraction $A$ ', we take its input storage allocation context to be $(l+1,0$, stat $)$, where $l$ is the scope nesting level of the surrounding action.

\section{Implementation}

The action transformer accepts an action (annotated with sort information), and returns a simplified action.

Algebraic simplification is implemented by a simple ML function, law. Given an (annotated) action $A$, law $A$ returns an equivalent but simplified action.

The more complicated transformations (transient and binding elimination, and static allocation) are implemented by ML functions simpAction and simp$Y$ ielder. Given an (annotated) action $A$, simpAction $A T B S$ returns a quadruple $\left(A^{\prime}, T^{\prime}, B^{\prime}, S^{\prime}\right)$, in accordance with judgment $(5) .{ }^{8}$ Similarly, simpYielder $Y T B S$ returns a transformed yielder $Y^{\prime}$, in accordance with judgment (9).

Here now is a complete worked example of action transformations. Consider the following Mini- $\triangle$ source program:

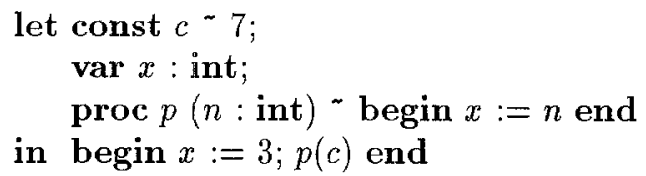

This is mapped by the parser, actioneer, and sort checker ${ }^{9}$ to the following program action:

\section{furthermore}

\footnotetext{
${ }^{8} \mathcal{K}$ corresponds to the sort information with which the action has been annotated.

9 The sort checker replaces failing actions by 'fail' and then algebraically simplifies the result.
} 




This program action is simplified by the transformer to the following:

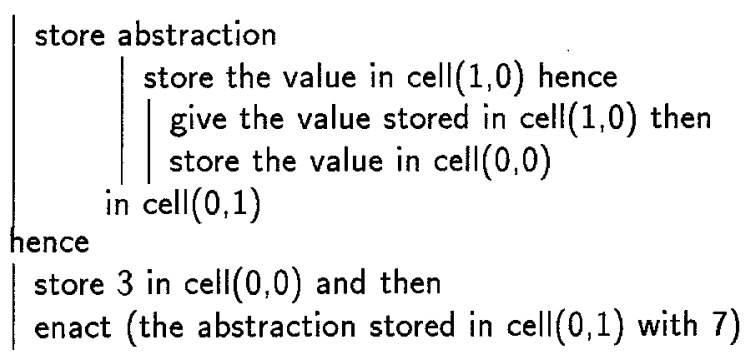

Note the complete elimination of bindings and of dynamic allocation. The transformed program action is about half the size of the original program action.

When the transformed program action is passed to the action notation code generator, inspection of the object code (not shown here) reveals that it is structurally very similar to the object code that would be generated by a simple hand-crafted compiler. It runs about twice as fast as the object code generated without action transformations.

Larger programs show larger speedups. The more declarations the program contains, the greater the benefit of binding elimination; and the more variables the program contains, the greater the benefit of static allocation. A small set of benchmarks [9] suggests that incorporating the action transformer improves generated compilers' object code from 15-100 times slower to only 7-30 times slower than the object code of hand-crafted compilers. (These figures exclude other code improvements, such as elimination of run-time sort checks, outside the scope of this paper.)

\section{Conclusion}

The transformations we have discussed in this paper are not the ones found in ordinary 'optimising' compilers. The need for these transformations is a consequence of generating compilers automatically from semantic descriptions. The 
need for binding elimination, for instance, is a consequence of the presence of bindings in the source language's semantic description. This factor applies not only to action semantics but also to denotational semantics and other formalisms.

Denotational semantics maps source programs to $\lambda$-notation, in which environments and stores are passed around like ordinary values. Sophisticated analysis is needed to detect rather basic properties such as storage single-threadedness and block structure [14, 15]. Environment elimination can be achieved by partial evaluation $[5,6]$. Any realistic denotational-semantics directed compiler generator must, however, impose some structure on the semantic descriptions it accepts. For example, the DML system [13] imposes the continuation-passing style, which guarantees storage single-threadedness.

We believe that action semantics is particularly well-suited to semanticsdirected compiler generation. Action notation has much more structure than $\lambda$-notation. The concept of bindings is built in, and bindings have definite scope; the concept of storage is also built in, and is guaranteed to be single-threaded. This structure gives action-semantics directed compiler generators a better handle on the problem than other compiler generators. Equally important, the good pragmatic qualities of action semantics make it feasible for the same semantic description to be used by language designers, implementors, and programmers - which is not true of other semantic formalisms!

Other important work on action-semantics directed compiler generation has been done by Palsberg [12], Ørbæk [11], and Doh [3]. Palsberg's compiler generator CANTOR is broadly similar to ACTRESS, but it restricts itself to staticallyscoped source languages. Nevertheless, the current version does not actually eliminate bindings. Ørbæk's system OAsIS includes an action notation code generator that incorporates a variety of forward and backward analyses, enabling it to eliminate many time-consuming run-time checks from the generated object code.

Doh's prototyping system, based on a category-sorted algebraic model for action semantics [4], extracts a binding-time semantics from an action-semantic description. It generates a syntax-directed translator that translates the source program to a program action annotated with binding-time information. This annotation will assist a static evaluator to identify which parts of the program action can be statically performed. As compared with Doh's method, it seems that our method can eliminate more bindings, and is applicable to a larger subset of action notation.

An ACTRESS-generated compiler cannot yet match the quality of a competent hand-crafted compiler. If the source language is statically scoped, the compiler writer will know that every applied occurrence of an identifier $I$ can be substituted, whereas the AcTRESS-generated compiler tests each applied occurrence individually to determine whether substitution is possible. Similarly, if the source language is block-structured, the compiler writer will know that variable declarations can be implemented by static allocation, whereas the ACTRESS-generated compiler tests each allocation individually to determine whether it is dynamic or static. 
The compiler writer acquires this knowledge by studying the source language's description, and inferring key properties such as whether the language is statically scoped and block structured. We postulate that a future version of ACTRESS will be able to infer such properties automatically from the actionsemantic description ${ }^{10}$, and will be able to build its knowledge of these properties into the generated compiler. In effect, it will transform the semantic equations (and hence the generated actioneer) at compiler generation time, rather than transform every program action at compilation time.

The program action serves as an intermediate representation of the source program's semantics. Of course, it is commonplace for hand-crafted compilers to use intermediate representations at the interface between their front and back ends [1]. Compared to these ad hoc intermediate representations, however, action notation has several important advantages:

- Action notation is independent of both source and target languages. Thus it can serve as a universal intermediate representation.

- Action notation is formally specified [7]. This will allow us (at least in principle) to prove the correctness of our transformations with respect to the formal semantics of the actions.

Another intriguing possibility is to express the standard compiler transformations - such as common subexpression elimination and code motion - in terms of action notation. This would allow us to formalise the standard transformations and (at least in principle) to prove their correctness. It would also have the practical benefit of enabling us to implement the standard transformations, once and for all, in the form of a module that could optionally be plugged into ACTRESS-generated compilers.

\section{Acknowledgments}

Deryck Brown, the third member of the ACTRESS group, has made innumerable comments and suggestions on the work reported here. Peter Mosses, Simon Peyton Jones, David Schmidt, and Phil Wadler have also, at various times, provided valuable contributions. We are happy to acknowledge them all.

\section{References}

1. A. Aho, R. Sethi, and J. Ullman. Compilers - Principles, Techniques, and Tools. Addison-Wesley, 1986.

2. D. F. Brown, H. Moura, and D. A. Watt. ActRess: an action semantics directed compiler generator. In U. Kastens and P. Pfahler, editors, Compiler Construction, 95-109. Springer-Verlag, 1992.

3. K.-G. Doh. Action semantics-directed prototyping. $\mathrm{PhD}$ thesis, Kansas State University, 1992 .

$\overline{10}$ We already know how to test for static-scopedness - see Section 4. 
4. S. Even and D. A. Schmidt. Category sorted algebra-based action semantics. Theoretical Computer Science, (77):73-96, 1990.

5. C. K. Gomard and N. D. Jones. A partial evaluator for the untyped lambdacalculus. Journal of Functional Programmming, 1(1):21-69, 1991.

6. N. D. Jones, C. K. Gomard, and P. Sestoft. Partial Evaluation and Automatic Program Generation. Prentice Hall International, 1993.

7. P. D. Mosses. Action Semantics. Cambridge University Press, 1992.

8. P. D. Mosses and D. A. Watt. The use of action semantics. In M. Wirsing, editor, Formal Description of Programming Concepts, 135-163. North Holland, 1987.

9. H. Moura. Action notation transformations. $\mathrm{PhD}$ thesis, University of Glasgow, 1993.

10. H. Moura and D. A. Watt. Action transformations in the ACTREss compiler generator. Report FM-94-02, Department of Computing Science, University of Glasgow, 1994.

11. P. Ørbæk. Analysis and optimisation of actions. MSc dissertation, Aarhus University, 1993.

12. J. Palsberg. Provably correct compiler generation. $\mathrm{PhD}$ thesis, Aarhus University, 1992.

13. M. Pettersson and P. Fritzson. DML - a meta-language and system for the generation of practical and efficient compilers from denotational specifications. In International Conference on Computer Languages, Oakland, California, 1992.

14. D. A. Schmidt. Detecting global variables in denotational specifications. $A C M$ Transactions on Programming Languages and Systems, 7(2):299-310, 1985.

15. D. A. Schmidt. Detecting stack-based environments in denotational definitions. Science of Computer Programming, 11:107-131, 1988.

16. D. A. Watt. Programming Language Syntax and Semantics. Prentice Hall International, 1991.

\section{A Action-Semantic Description of MINI- $\triangle$}

The following are extracts from the action-semantic description of MiNI- $\triangle$. Only those parts of it referred to in the paper are shown.

\section{A.1 Semantic Entities}

(1) value $=$ truth-value $\mid$ integer .

(2) argument $=$ value $\mid$ cell .

(3) bindable $=$ value $\mid$ cell $\mid$ abstraction .

(4) storable $=$ value

\section{A.2 Semantic Functions}

- execute _ .: Command $\rightarrow$

action [storing] [using current bindings | current storage] .

(1) execute $\llbracket I^{\prime \prime}:=" E \rrbracket=$

evaluate $E$ then store the value in the cell bound to $I$. 
(2) execute 【I:Identifier "(" A:Actual ")" \= give-argument $A$ then

enact (the abstraction bound to $I$ with the argument) .

(3) execute $\llbracket C_{1} " ; " C_{2} \rrbracket=$ execute $C_{1}$ and then execute $C_{2}$.

(4) execute 【 "let" $D$ :Declaration "in" $C$ :Command 》= furthermore elaborate $D$ hence execute $C$.

- evaluate - : Expression $\rightarrow$ action [giving a value] [using current bindings | current storage]

(5) evaluate $N:$ Numeral $=$ give integer-valuation $N$.

(6) evaluate $I$ :Identifier $=$ give the value bound to $I$ or give the value stored in the cell bound to $I$.

- elaborate _ :: Declaration $\rightarrow$

action [binding | storing] [using current bindings | current storage] .

(7) elaborate [ "const" $I$ :Identifier " " $E$ :Expression 》 = evaluate $E$ then bind $I$ to the value.

(8) elaborate [ "var" $I$ ":" $T \rrbracket=$ allocate a cell then bind $I$ to the cell .

(9) elaborate [ "proc" $I$ :Identifier "(" $F$ :Formal ")" " $C$ :Command 》 = recursively bind $I$ to closure abstraction

(10) elaborate [ $D_{1}$ :Declaration ";" $D_{2}:$ Declaration 》 = furthermore bind-parameter $F$ hence execute $C$.

elaborate $D_{1}$ before elaborate $D_{2}$.

- bind-parameter - :: Formal $\rightarrow$ action [binding] .

(11) bind-parameter $\llbracket I$ :Identifier ":" $T:$ Type $\rrbracket=$ bind $I$ to the value .

- give-argument _ :: Actual $\rightarrow$ action [giving an argument] [using current bindings I current storage] .

(12) give-argument $\llbracket E$ :Expression $\rrbracket=$ evaluate $E$. 